\title{
Reduction of blood eosinophil counts in eosinophilic chronic rhinosinusitis after surgery
}

\author{
A HONMA, D TAKAGI, Y NAKAMARU, A HOMMA, M SUZUKI, S FUKUDA \\ Department of Otolaryngology - Head and Neck Surgery, Hokkaido University Graduate School of Medicine, \\ Sapporo, Japan
}

\begin{abstract}
Objective: This study aimed to predict eosinophilic chronic rhinosinusitis prognosis by investigating changes in the blood eosinophil count and other disease biomarkers after surgery.

Methods: Blood eosinophil numbers and serum interleukin-5 levels were measured in 22 eosinophilic chronic rhinosinusitis patients before and after functional endoscopic sinus surgery, and compared with equivalent measures in non-eosinophilic chronic rhinosinusitis patients and chronic rhinosinusitis without polyps patients. Differences between well-controlled eosinophilic chronic rhinosinusitis patients and those who experienced recurrence were also assessed.

Results: Blood eosinophil numbers and serum interleukin-5 level decreased after surgery in eosinophilic chronic rhinosinusitis patients. In this patient group, blood eosinophil counts before surgery were significantly higher in patients who experienced recurrence $(825.7 \pm 26.1$ vs $443.9 \pm 76.6$ cells $/ \mu 1, p<0.05)$, and decreased significantly after surgery $(825.7 \pm 26.1$ vs $76.7 \pm 25.8$ cells $/ \mu 1, p<0.05)$.

Conclusion: Blood eosinophil numbers may reflect disease severity in eosinophilic chronic rhinosinusitis patients and their prognosis after surgery.
\end{abstract}

Keywords: Sinusitis; Eosinophilia; Recurrence

\section{Introduction}

Chronic rhinosinusitis is one of the most frequently encountered diseases in the field of otolaryngology and is classified into two subgroups: with and without nasal polyps. Chronic rhinosinusitis without nasal polyps is more likely to be caused by a bacterial infection, whereas chronic rhinosinusitis with nasal polyps is more likely to present with blood eosinophilia and asthma. ${ }^{1-4}$

Recently, there have been increasing numbers of intractable chronic rhinosinusitis cases, presenting with nasal polyps and high levels of eosinophil infiltration. This so-called eosinophilic chronic rhinosinusitis subtype commonly coexists with asthma (including aspirin-intolerant asthma) and frequently recurs after surgery. Histopathological studies have demonstrated that the inflammation involves accumulation of activated eosinophils in the sinus mucosa. ${ }^{5}$ These eosinophils are reported to play a role in the pathogenesis of chronic rhinosinusitis via releasing granule proteins such as eosinophil cationic protein. ${ }^{6}$

The relationship between chronic rhinosinusitis and asthma is well established: an asthma prevalence of 50 per cent is reported in chronic rhinosinusitis patients, and asthmatic symptoms recover after medical and/or surgical treatment in 60-88 per cent of patients. ${ }^{7}$ Eosinophil infiltration is one of the histological features shared by asthma and chronic rhinosinusitis with nasal polyps, but little is known about the mechanisms underlying eosinophilic inflammation in chronic rhinosinusitis, and particularly in eosinophilic chronic rhinosinusitis.

Management after functional endoscopic sinus surgery (FESS) is important, and an elevated peripheral eosinophil count before surgery is considered an important predictor of chronic rhinosinusitis recurrence after FESS. ${ }^{8}$ However, changes in the blood eosinophil count after surgery have not been reported.

This study evaluated blood eosinophil counts and the levels of other biomarkers before and after surgery to try to identify novel prognosis factors for eosinophilic chronic rhinosinusitis.

\section{Materials and methods}

Patients

This study evaluated 78 chronic rhinosinusitis patients who underwent FESS at the Department of Otolaryngology - Head and Neck Surgery, Hokkaido 
University Hospital, between April 2007 and December 2012. Patients with chronic rhinosinusitis resulting from a paranasal sinus tumour or paranasal sinus cysts developing after surgery were excluded. The patient cohort comprised 33 women (42 per cent) and 45 men (58 per cent) with a mean age of $52.8 \pm 1.52$ years (range 13-83 years).

Chronic rhinosinusitis was diagnosed based on clinical symptoms (anterior and/or posterior nasal drip, nasal obstruction, decreased sense of smell) lasting for more than 12 weeks, nasal endoscopy and computed tomography (CT) imaging of the paranasal cavities. ${ }^{9}$ Chronic rhinosinusitis patients were classified into chronic rhinosinusitis with or without nasal polyps groups based on the presence or absence of bilateral polyps, respectively, as confirmed by endoscopy and CT imaging. Furthermore, chronic rhinosinusitis with nasal polyps was subdivided into eosinophilic and non-eosinophilic chronic rhinosinusitis according to the degree of eosinophilic infiltration into sinus mucosal tissue (Figure 1). ${ }^{8}$

Before surgery, all patients underwent CT imaging and the peripheral eosinophil count, total serum immunoglobulin E (IgE) and respiratory function were measured. Computed tomography scans were evaluated by an otolaryngologist and a radiologist who were blinded to the disease subtype and staged according to the Lund-MacKay scoring system. ${ }^{9}$

This study was approved by the Institutional Review Board of Hokkaido University Hospital for Clinical Research (approval number 012-0399), and written informed consent was obtained from all patients.

\section{Evaluation of tissue and blood eosinophil counts}

Sinus mucosal tissue samples were collected from the ethmoid cavity during FESS followed by hematoxylin and eosin staining to detect tissue eosinophilia. The number of eosinophils in each tissue sample was

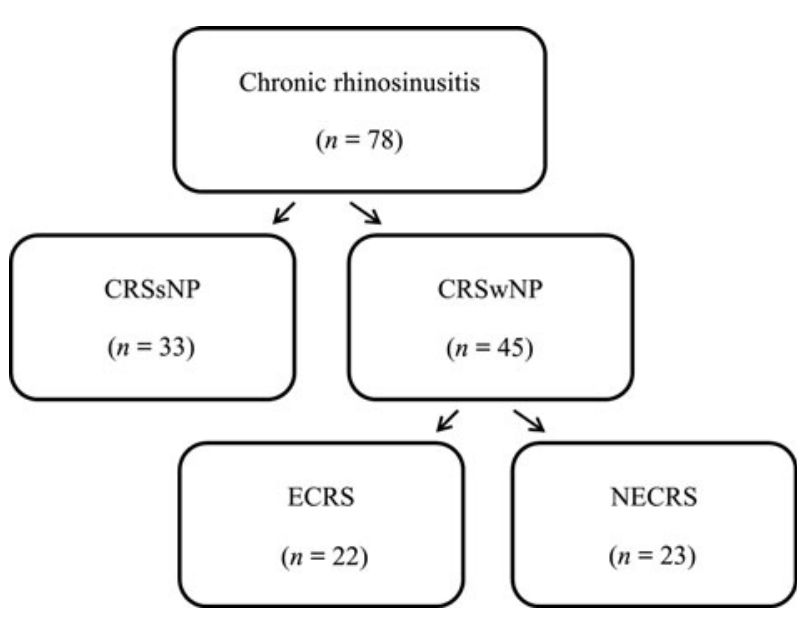

FIG. 1

Flowchart showing patient classification in the study. CRSsNP = chronic rhinosinusitis without nasal polyps; CRSwNP = chronic rhinosinusitis with nasal polyps; NECRS = non-eosinophilic chronic rhinosinusitis; ECRS = eosinophilic chronic rhinosinusitis counted in a single high-power magnification field ( $\times 400$ magnification, $0.307 \mathrm{~mm}^{2}$ ), and counts from three fields in the mucosal area were averaged. The disease was subclassified according to the degree of eosinophilic infiltration (average number of tissue eosinophils) into eosinophilic chronic rhinosinusitis (at least 120 cells per high-power magnification field) and non-eosinophilic chronic rhinosinusitis group (less than 120 cells per high-power magnification field). ${ }^{8}$ The Blood eosinophil count and total serum $\mathrm{IgE}$ level were measured before FESS. Blood eosinophil counts and IgE levels before and after FESS were available for 13 eosinophilic chronic rhinosinusitis patients.

\section{Measurement of interleukin-5 levels}

Serum samples were collected before and after surgery, and stored at $-20{ }^{\circ} \mathrm{C}$ for analysis. Interleukin-5 (IL-5) was assayed using a commercial enzyme-linked immunosorbent assay kit (Hu IL-5 ELISA; BioSource International, Camarillo, California, USA) according to the manufacturer's instructions, including the use of standard curves and controls.

\section{Statistical analysis}

Data are expressed as means, standard error of the mean and range. Fisher's exact test was used to compare the sex ratio, asthma prevalence and nasal symptoms. Oneway analysis of variance followed by post-hoc testing was used to analyse the CT score, serum and tissue eosinophil counts, serum IgE levels, and respiratory function (percentage vital capacity; percentage forced expiratory volume in 1 second; forced expiratory flow at 50 per cent vital capacity $\div$ forced expiratory flow at 25 per cent vital capacity). The reduction in eosinophil count after surgery was compared between groups using paired $t$-tests. A $p$ value of less than 0.05 was considered statistically significant.

\section{Results}

\section{Clinical features of patients}

Of the 78 chronic rhinosinusitis patients, 45 (58 per cent) were diagnosed with chronic rhinosinusitis with nasal polyps and 33 (42 per cent) with chronic rhinosinusitis without nasal polyps. Of the 45 chronic rhinosinusitis with nasal polyps patients, 22 (49 per cent) were women, and 23 (51 per cent) were men, with a mean age of $52.7 \pm 1.7$ years (range 19-75 years). Twentysix chronic rhinosinusitis with nasal polyps patients (58 per cent) presented with concomitant asthma (Table I). The mean blood eosinophil count was $343 \pm 62.3$ cells $/ \mu \mathrm{l}(6.71 \pm 0.71$ per cent $)$.

Of the 45 chronic rhinosinusitis with nasal polyps patients, 22 (49 per cent) were classified as having eosinophilic chronic rhinosinusitis and 23 (51 per cent) as having non-eosinophilic chronic rhinosinusitis. The asthma prevalence (77 per cent vs 39 per cent; Table I) and blood eosinophil counts were significantly 


\begin{tabular}{|c|c|c|c|}
\hline \multicolumn{4}{|c|}{$\begin{array}{c}\text { TABLE I } \\
\text { CLINICAL PROFILES OF THE STUDY GROUPS }\end{array}$} \\
\hline \multirow[t]{2}{*}{ Characteristic } & \multirow[t]{2}{*}{ CRSsNP } & \multicolumn{2}{|c|}{ CRSwNP } \\
\hline & & NECRS & ECRS \\
\hline Total $(n)$ & 33 & 23 & 22 (RG 4, WG 18) \\
\hline Age (years), mean (range) & $53.1(13-83)$ & $51.5(19-70)$ & $54(26-75)$ \\
\hline Sex (female $/$ male $)$ & $11 / 22$ & $7 / 16$ & $15 / 7^{*}$ \\
\hline Asthma & $2 / 33$ & $9 / 23$ & $17 / 22^{*}$ \\
\hline CT score & $4.5 \pm 0.56$ & $14.9 \pm 1.34$ & $18.4 \pm 1.34$ \\
\hline Blood eosinophils (cells $/ \mu \mathrm{l})$ & $193 \pm 23.6$ & $402 \pm 123.1$ & $516 \pm 69.5^{*}$ \\
\hline Blood eosinophils ${ }^{\dagger}(\%)$ & $2.74 \pm 0.39$ & $5.39 \pm 0.98$ & $8.57 \pm 1.08^{*}$ \\
\hline$\% \mathrm{VC}$ & $117.8 \pm 2.68$ & $112.8 \pm 4.39$ & $114.4 \pm 3.68$ \\
\hline FEV $1 \%$ & $76.0 \pm 1.42$ & $73.2 \pm 2.61$ & $72.6 \pm 2.24$ \\
\hline V50/V25 & $4.00 \pm 0.29$ & $3.18 \pm 0.22$ & $4.08 \pm 0.22$ \\
\hline
\end{tabular}

${ }^{*} p<0.05 .{ }^{\dagger}$ As a percentage of white blood cells. Data are mean \pm standard error, except where otherwise indicated. CRSsNP $=$ chronic rhinosinusitis without nasal polyps; CRSwNP = chronic rhinosinusitis with nasal polyps; NECRS = non-eosinophilic chronic rhinosinusitis; ECRS = eosinophilic chronic rhinosinusitis; $\mathrm{RG}=$ recurrence group; $\mathrm{WG}=$ well-controlled group $; \mathrm{CT}=$ computed tomography; $\% \mathrm{VC}=$ percentage vital capacity; FEV $1 \%$ = percentage forced expiratory volume in 1 second; V50/V25 = forced expiratory flow at 50 per cent vital capacity $\div$ forced expiratory flow at 25 per cent vital capacity

higher in eosinophilic chronic rhinosinusitis patients than in non-eosinophilic chronic rhinosinusitis patients. There were no significant between-group differences in age, serum IgE level or any factor related to respiratory function.

\section{Reduced blood eosinophil count after surgery in eosinophilic chronic rhinosinusitis patients}

Twenty-four chronic rhinosinusitis with nasal polyps patients were followed for over one year and up to five years after surgery. Blood eosinophil counts had significantly decreased at six months after surgery in eosinophilic chronic rhinosinusitis patients $(n=13$; before surgery, $517.9 \pm 104.2$ cells $/ \mu \mathrm{l}$; after surgery, $291.2 \pm$ 53.9 cells $/ \mu \mathrm{l} ; p<0.05$; Figure 2 ), but not in non-eosinophilic chronic rhinosinusitis patients (before surgery, $311.8 \pm 54.9$ cells $/ \mu \mathrm{l} ;$ after surgery, $311.0 \pm 72.5$

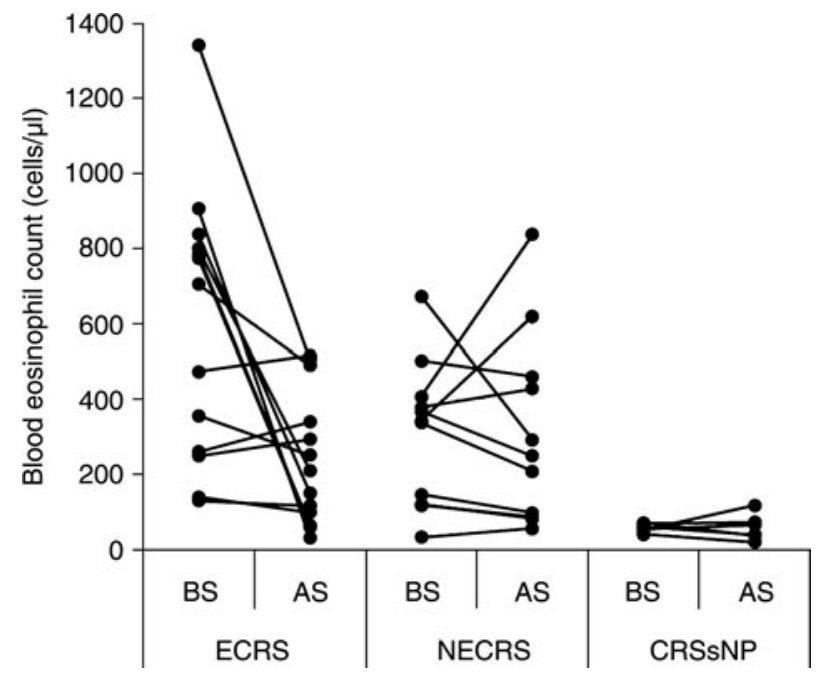

FIG. 2

Graph showing the blood eosinophil count before (BS) and six months after (AS) surgery in eosinophilic chronic rhinosinusitis (ECRS), non-eosinophilic chronic rhinosinusitis (NECRS) and chronic rhinosinusitis without nasal polyps (CRSsNP) patients. cells $/ \mu 1)$. No decrease in blood eosinophil count was observed in chronic rhinosinusitis without nasal polyps patients (before surgery, $57.8 \pm 4.4$ cells $/ \mu \mathrm{l}$; after surgery, $58.7 \pm 12.9$ cells $/ \mu \mathrm{l})$. Serum $\mathrm{IgE}$ levels also tended to be reduced after surgery in both groups, but the difference was not significant.

Serum IL-5 levels also tended to be reduced after surgery in eosinophilic chronic rhinosinusitis patients, but the difference was not significant. However, the serum IL-5 ratio (before FESS $\div$ after FESS) was significantly higher in eosinophilic chronic rhinosinusitis patients than in chronic rhinosinusitis without nasal polyps patients (Table II).

\section{Severe reduction in eosinophils in patients experiencing recurrence}

All eosinophilic chronic rhinosinusitis patients and those non-eosinophilic chronic rhinosinusitis patients suffering from asthma were treated with oral prednisolone (5-10 mg/day) for one week after FESS. Blood eosinophil numbers were counted at least one month after cessation of oral prednisolone treatment. All chronic rhinosinusitis with nasal polyps patients were

\begin{tabular}{lccc}
\multicolumn{4}{c}{ TABLE II } \\
REDUCTION IN SERUM INTERLEUKIN-5 LEVELS AFTER \\
SURGERY \\
\hline Patient group & $\begin{array}{c}\text { Serum IL-5 concentration } \\
(\mathrm{pg} / \mathrm{ml})\end{array}$ & Serum IL-5 ratio \\
\cline { 2 - 3 } & \multicolumn{2}{c}{ Before FESS } & After FESS \\
\hline CRSsNP & $22.9 \pm 6.9$ & $30.3 \pm 2.3$ & $0.65 \pm 0.14$ \\
CRSwNP & & & \\
- ECRS & $44.3 \pm 4.6$ & $32.6 \pm 7.7$ & $1.48 \pm 0.19^{*}$ \\
- NECRS & $46.9 \pm 5.9$ & $46.2 \pm 6.7$ & $1.02 \pm 0.14$ \\
\hline
\end{tabular}

${ }^{*} p<0.05$ compared with the CRSsNP group. IL-5 = interleukin-5; FESS = functional endoscopic sinus surgery; CRSsNP = chronic rhinosinusitis without nasal polyps; CRSwNP $=$ chronic rhinosinusitis with nasal polyps; NECRS $=$ non-eosinophilic chronic rhinosinusitis; ECRS = eosinophilic chronic rhinosinusitis 
treated with a steroid nasal spray. Eighteen of the 22 eosinophilic chronic rhinosinusitis patients had no recurrence (well-controlled group) and 4 (18 per cent) experienced nasal polyp recurrence (recurrence group; requiring systemic steroid treatment) during follow up (1-5 years; Table I). Blood eosinophil counts were significantly higher in the recurrence group than in the well-controlled group $(825.7 \pm 26.1$ cells $/ \mu 1$ vs $443.9 \pm 76.6$ cells $/ \mu$; Table I; Figure 3). Mucosal eosinophil counts were also higher in the recurrence group than in the well-controlled group, but the difference was not significant $(594.7 \pm 117.2$ vs $365.1 \pm$ 47.3 cells per high-power magnification field; Figure 3).

Furthermore, in the recurrence group, the blood eosinophil count decreased significantly after FESS ( $n=4$; before surgery, $825.7 \pm 26.1$ cells $/ \mu$; after surgery, $76.7 \pm 25.8$ cells $/ \mu \mathrm{l} ; p<0.05$; Figure $4 \mathrm{a}$ ). In the well-controlled group, the blood eosinophil count also decreased after FESS, but the difference was not significant $(n=9$; before surgery, $495.4 \pm$ 124.2 cells $/ \mu \mathrm{l}$; after surgery, $313.8 \pm 50.8$ cells $/ \mu \mathrm{l}$ ). The blood eosinophil ratio (before FESS $\div$ after FESS) in eosinophilic chronic rhinosinusitis patients was significantly higher in the recurrence group than in the well-controlled group $(11.33 \pm 1.85$ vs $1.79 \pm$ 0.80; Figure 4b). However, in the recurrence group, the blood eosinophil count increased to $705.0 \pm 86.0$ (median 512 cells $/ \mu \mathrm{l}$ ) during recurrence (at 8 months to 2 years after surgery; median 1 year).

\section{Discussion}

The most important finding in this study was the significant decrease in blood eosinophil count after FESS in eosinophilic chronic rhinosinusitis patients. Moreover, among eosinophilic chronic rhinosinusitis patients, those with a significantly reduced blood eosinophil count after FESS were more likely to experience chronic rhinosinusitis recurrence.

Eosinophilic chronic rhinosinusitis is histologically characterised by marked eosinophil infiltration into the nasal polyps and sinus mucosa that is refractory to conventional therapy and readily recurs after surgery: there is also a high prevalence of asthma, including aspirinintolerant asthma. ${ }^{1}$ Mucosal eosinophilia (defined as more than 120 cells per high-power magnification

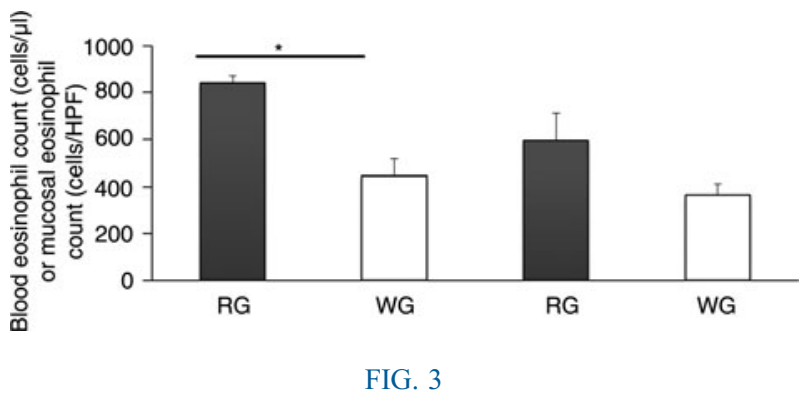

Histogram showing blood eosinophil counts in eosinophilic chronic rhinosinusitis patients in the recurrence $(R G)$ and well-controlled (WG) groups. HPF $=$ high-power magnification field. ${ }^{*} p<0.05$.

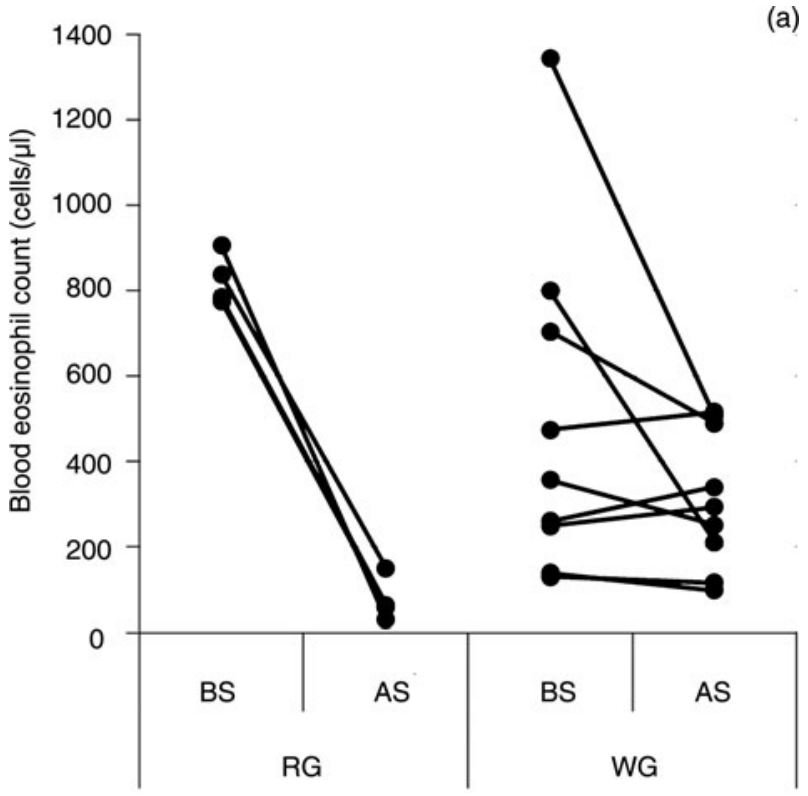

(b)

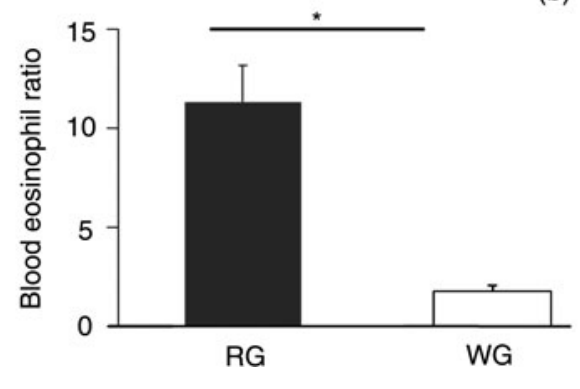

FIG. 4

Graphs showing (a) blood eosinophil counts before (BS) and after (AS) surgery and (b) the blood eosinophil ratio in eosinophilic chronic rhinosinusitis patients in the recurrence $(\mathrm{RG})$ and well-controlled (WG) groups. ${ }^{*} p<0.05$.

field) is reported to most strongly correlate with chronic rhinosinusitis recurrence. ${ }^{8}$

The association of eosinophilic chronic rhinosinusitis with asthma is widely accepted..$^{10}$ The present study also found a higher prevalence of asthma in eosinophilic chronic rhinosinusitis patients.

As expected, the blood eosinophil count was higher in eosinophilic chronic rhinosinusitis patients than in noneosinophilic chronic rhinosinusitis patients. Eosinophil accumulation and activation are enhanced by IL-5 and eotaxin overproduction in nasal polyp tissue. ${ }^{11}$ Interleukin-5 appears to play a role in eosinophil activation and survival. IL-5 appears to play a role in the activation and survival of eosinophils. Chronic rhinosinusitis with nasal polyps patients have higher mucosal IL-5 protein levels compared with chronic rhinosinusitis without nasal polyps patients. ${ }^{12}$ Moreover, in chronic rhinosinusitis with nasal polyps patients, IL-5 protein levels are reported to be higher in allergic nasal polyps compared with non-allergic nasal polyps. ${ }^{13}$ The present study found a significant decrease in blood eosinophils in eosinophilic chronic rhinosinusitis patients after surgery, along with a reduction in serum IL-5 level. 
Thus, the decreased IL-5 levels and eosinophil numbers in nasal polyps after FESS might lead to decreased serum IL-5 levels and blood eosinophil counts.

In the present study, four eosinophilic chronic rhinosinusitis patients experienced recurrence after surgery, and blood eosinophil counts were higher in the recurrence group than in the well-controlled group. Interestingly, the blood eosinophil count was significantly reduced in the recurrence group after surgery. Furthermore, some patients in the well-controlled group had only a moderate reduction in eosinophil count after surgery, even though their blood eosinophil count was high before surgery. The cause of this is unclear; however, all four patients in the recurrence group suffered from asthma and local eosinophilia. Among eosinophilic chronic rhinosinusitis patients, those with recurrence are reported to have significantly higher mucosal eosinophil and blood eosinophil counts compared with well-controlled patients. ${ }^{9}$ It is possible that the removal of polyps (which include toxic products from eosinophils and high IL-5 concentrations) in severe eosinophilic chronic rhinosinusitis patients might decrease the serum IL-5 level, leading to reduced peripheral eosinophil numbers. Several reports indicate that increased cysteinyl leukotriene receptor levels in eosinophilic chronic rhinosinusitis patients intensify eosinophilic inflammation in the paranasal mucosa. ${ }^{14-16}$ Eosinophilic chronic rhinosinusitis patients with accompanying aspirin-intolerant asthma are reported to have elevated cysteinyl leukotriene production. ${ }^{14}$ Higashi et al. reported that surgical resection of both large nasal polyps and extensive hyperplastic sinonasal mucosa helps to halt the vicious cycle of cysteinyl leukotriene and chemotactic factor production in sinonasal tissue. ${ }^{17}$ Restoration of blood eosinophil counts accompanied rhinosinusitis recurrence. Recently, natural killer $\mathrm{T}$ cells were reported to release IL-33 during viral infection, which enhances IL-5 production and subsequently leads to eosinophil accumulation. ${ }^{18}$ Eosinophil infiltration from the epithelium to the sinus cavity might be one mechanism responsible for the recurrence of eosinophilic chronic rhinosinusitis.

- Blood eosinophil counts decreased significantly after functional endoscopic sinus surgery in eosinophilic chronic rhinosinusitis patients

- Blood eosinophil counts before surgery were significantly higher in eosinophilic chronic rhinosinusitis patients who suffered recurrence

- Blood eosinophil counts were restored to presurgery levels in patients suffering recurrence

- Pre-surgery blood eosinophil counts might be a prognostic indicator in eosinophilic chronic rhinosinusitis
Functional endoscopic sinus surgery is proposed to have the beneficial effect of reducing eosinophil infiltration into the sinus mucosa by decreasing the endothelial L-selectin ligand level. ${ }^{19}$ It is important to note that surgical treatment alone does not cure nasal mucosa disease in eosinophilic chronic rhinosinusitis patients: supplementary medical treatment and longterm follow up are essential.

\section{Conclusion}

Blood eosinophil counts are decreased in eosinophilic chronic rhinosinusitis patients after FESS, especially in patients who go on to suffer recurrence. Therefore, reduced blood eosinophil numbers may be a prognostic factor in eosinophilic chronic rhinosinusitis and blood eosinophil counts might be useful for determining treatment after surgery. However, further studies in a larger patient cohort are necessary to support this finding.

\section{Acknowledgements}

We are grateful to Y Ishida for her expert technical assistance. This study was supported by a Grant-in-Aid for Scientific Research type (C).

\section{References}

1 Meltzer EO, Hamilos DL, Hadley JA, Lanza DC, Marple BF, Nicklas RA et al. Rhinosinusitis: establishing definitions for clinical research and patient care. J Allergy Clin Immunol 2004;114:155-212

2 Ichimura K, Shimazaki Y, Ishibashi T, Higo R. Effect of new macrolide roxithromycin upon nasal polyps associated with chronic sinusitis. Auris Nasus Larynx 1996;23:48-56

3 Moriyama H, Yanagi K, Ohtori N, Fukami M. Evaluation of endoscopic sinus surgery for chronic sinusitis: post-operative erythromycin therapy. Rhinology 1995;33:166-70

4 Soler ZM, Sauer DA, Mace J, Smith TL. Relationship between clinical measures and histopathologic findings in chronic rhinosinusitis. Otolaryngol Head Neck Surg 2009;141:454-61

5 Harlin SL, Ansel DG, Lane SR, Myers J, Kephart GM, Gleich GJ. A clinical and pathological study of chronic sinusitis: the role of the eosinophil. J Allergy Clin Immunol 1988;81:867-75

6 Motojima S, Frigas E, Loegering DA, Gleich GJ. Toxicity of eosinophil cationic proteins for guinea pig tracheal epithelium in vitro. Am Rev Respir Dis 1989;139:801-5

7 Slavin RG. Complications of allergic rhinitis: Implications for sinusitis and asthma. J Allergy Clin Immunol 1988;101:357-60

8 Matsuwaki Y, Ookushi T, Asaka D, Mori E, Nakajima T, Yoshida T et al. Chronic rhinosinusitis: risk factors for the recurrence of chronic rhinosinusitis based on 5-year follow-up after endoscopic sinus surgery. Int Arch Allergy Immunol 2008; 146(suppl 1):77-81

9 Lund VJ, Kennedy DW. Staging for rhinosinusitis. Otolaryngol Head Neck Surg 1997;117:S35-40

$10 \mathrm{Hu} \mathrm{Y,} \mathrm{Cao} \mathrm{PP,} \mathrm{Liang} \mathrm{GT,} \mathrm{Cui} \mathrm{YH,} \mathrm{Liu} \mathrm{Z.} \mathrm{Diagnostic} \mathrm{signifi-}$ cance of blood eosinophil count in eosinophilic chronic rhinosinusitis with nasal polyps in Chinese adults. Laryngoscope 2012; 122:498-503

11 Van Zele T, Claeys S, Gevaert P, Van Maele G, Holtappels G, Van Cauwenberge $\mathrm{P}$ et al. Differentiation of chronic sinus diseases by measurement of inflammatory mediators. Allergy 2006;61:1280-9

12 Sejima T, Holtappels G, Kikuchi H, Imayoshi S, Ichimura K, Bachert C. Cytokine profiles in Japanese patients with chronic rhinosinusitis. Allergol Int 2012;61:115-22

13 Hamilos DL, Leung DY, Huston DP, Kamil A, Wood R, Hamid Q. GM-CSF, IL-5 and RANTES immunoreactivity and mRNA expression in chronic hyperplastic sinusitis with nasal polyposis (NP). Clin Exp Allergy 1998;28:1145-52 
14 Steinke JW, Bradley D, Arango P. Cysteinyl leukotriene expression in chronic hyperplastic sinusitis-nasal polyposis: Importance to eosinophilia and asthma. J Allergy Clin Immunol 2003;112: 342-9

15 Sousa AR, Parikh A, Scadding G, Corrigan CJ, Lee TH. Leukotriene-receptor expression on nasal mucosal inflammatory cells in aspirin-sensitive rhinosinusitis. N Engl J Med 2002;347: 1493-9

16 Shirasaki H, Kanaizumi E, Watanabe K, Matsui T, Sato J, Narita $\mathrm{S}$ et al. Expression and localization of the cysteinyl leukotriene 1 receptor in human nasal mucosa. Clin Exp Allergy 2002;32: 1007-12

17 Higashi N, Taniguchi M, Mita H, Kawagishi Y, Ishii T, Higashi A et al. Clinical features of asthmatic patients with increased urinary leukotriene E4 excretion (hyperleukotrienuria): Involvement of chronic hyperplastic rhinosinusitis with nasal polyposis. J Allergy Clin Immunol 2004;113:277-83

18 Gorski SA, Hahn YS, Braciale TJ. Group 2 innate lymphoid cell production of IL-5 is regulated by NKT cells during influenza virus infection. PLoS Pathog 2013;9:e1003615
19 Myller JP, Toppila-Salmi SK, Toppila EM, Torkkeli TV, Numminen JE, Renkonen RL et al. Mucosal eosinophils and l-selectin ligands are associated with invasive and noninvasive sinus surgery outcomes. Am J Respir Crit Care Med 2005; 171:1350-7

Address for correspondence:

Dr D Takagi,

North 15, West 7, Kita-ku,

Sapporo 060-8638, Japan

Fax: +81 117177566

E-mail: daitamed@huhp.hokudai.ac.jp

Dr D Takagi takes responsibility for the integrity of the content of the paper

Competing interests: None declared 